\title{
Interactive comment on "Particulate matter (PM) episodes at a suburban site in Hong Kong: evolution of PM characteristics and role of photochemistry in secondary aerosol formation" by Yi Ming Qin et al.
}

\section{Anonymous Referee \#1}

Received and published: 28 July 2016

The manuscript of Y. M. Qin et al. presents the characteristics of fine PM in Hong Kong (chemical composition, size distribution, degrees of oxygenation, photochemical production and organic mass spectra) based on high resolution time-of-flight aerosol mass spectrometer (HR-ToF-AMS) measurements, positive matrix factorization (PMF) and air mass back-trajectory analyses. The manuscript is well structured and presented in a clear way. The methods used (PMF, back-trajectory analysis) are not innovative but are valid and well applied. The conclusions of this manuscript (e.g. the influence of meteorological conditions, particle mixing state, and the importance of photochemical aging on SOA formation in areas with high PM concentrations) are of scientific sig- 
nificance. I therefore recommend publishing this work in Atmospheric Chemistry and Physics after the authors respond to the following comments.

Specific comments: 1. Page 3, Line 47-49: it should be more careful for the statement that SVOOA is a proxy for fresh SOA while LVOOA is aged SOA. There isn't an absolute relationship between the "fresh" / the "aged" (terms involved in the OA formation and evolution) and the volatility / oxidation state (terms for OA physicochemical properties). The description in Page 8744 of Li et al. (2013) could be considered to be adopted in this study: "less-oxidized OA (e.g. SVOOA)" and "more-oxidized OA (e.g. LVOOA)". Other statements about "fresh" SOA (SVOOA) and "aged" SOA (LVOOA) in other parts of the text (e.g. abstract) are suggested to be adjusted.

2. Page 9, Line 192-193: "aged" is an ambiguous term and the ageing process includes several specific mechanisms. I suggest that "aged" is replaced by more specific descriptions. In addition, as you stated that small particle proportions are higher in both LWC and IR episodes, are the underlying reasons/mechanisms the same for both LWC and IR episodes? More specific discussion is suggested to replace "stronger local influences".

3. Page 9, Line 194-197: The accuracy of the derived particle mixing state from AMS is suggested to be discussed with comparing with single-particle instruments. Line 211: comparison with previous studies about the mixing state in Hong Kong will be helpful.

4. Page 10, Line 213-217: How those percentage numbers are calculated for the size variations? Line 219-220: How do you derive this result that "Since the particles were fresher and smaller during the days before episodes, they grew more rapidly as gas phase semi-volatile components condensed on the particles"? Is condensation the only contributor to the particle growth? Line 220-221: Please show evidence to support "the number concentrations of the pre-existing particles were lower before than during the episodes". Why the size variations for the LRT episodes are overlooked? What is your object to show the sizes variations? What are the implications? 
5. Page 10, Section 3.4 could be merged into Section 3.1 or just after the Section 3.1 (moving Section 3.2 to 3.3...).

ACPD

6. Page 12, Line 275: Please replace Figure 9c by Figure 9a.

Interactive comment on Atmos. Chem. Phys. Discuss., doi:10.5194/acp-2016-414, 2016.

Interactive

comment 